\title{
The Zeldovich \& Adhesion approximations and applications to the local universe
}

\author{
Johan Hidding $^{1}$, Rien van de Weygaert ${ }^{1}$ and Sergei Shandarin ${ }^{2}$ \\ ${ }^{1}$ Kapteyn Astronomical Institute, University of Groningen, \\ Postbus 800, NL-9700AV, Groningen, the Netherlands \\ email: johannes.hidding@gmail.com \\ ${ }^{2}$ Department of Physics and Astronomy, University of Kansas, \\ 1082 Malott,1251 Wescoe Hall Dr., Lawrence, KS 66045-7582
}

\begin{abstract}
The Zeldovich approximation (ZA) predicts the formation of a web of singularities. While these singularities may only exist in the most formal interpretation of the ZA, they provide a powerful tool for the analysis of initial conditions. We present a novel method to find the skeleton of the resulting cosmic web based on singularities in the primordial deformation tensor and its higher order derivatives. We show that the $A_{3}$-lines predict the formation of filaments in a two-dimensional model. We continue with applications of the adhesion model to visualise structures in the local $(z<0.03)$ universe.
\end{abstract}

\section{The Zeldovich approximation}

The Zeldovich Approximation (ZA) (Zeldovich 1970, Shandarin \& Zeldovich 1989) describes structure formation in the form of a deceptively simple equation

$$
\boldsymbol{x}(\boldsymbol{q}, t)=\boldsymbol{q}-D_{+}(t) \boldsymbol{\nabla} \Phi_{0}(\boldsymbol{q}) .
$$

Rather than describing just ballistic motion, this equation hides a formalism of Lagrangian collision-free fluid mechanics. In the context of emerging interest in phase-space folding descriptions of structure formation (Shandarin et al. 2012, Falck et al. 2012, Abel et al. 2012), it becomes all the more relevant to understand this expression at a much deeper level. We can see why there is more to the ZA than inertial motion, if we compute particle densities from the above expression. Density increases or decreases locally as a fluid element contracts or expands. Taking a fluid element from Lagrangian location $\boldsymbol{q}$, we can quantify its deformation in terms of the deformation tensor $d_{i j}=-\partial_{i} \partial_{j} \Phi_{0}$. This tensor is best studied locally in the eigenvector frame of reference $\left\{\boldsymbol{e}_{\lambda}\right\}$, where $d_{i j}$ becomes diagonal. The density is then

$$
\delta(\boldsymbol{x})+1=\sum_{q \in\left\{q^{\star}\right\}}\left|\operatorname{det} \frac{\partial x_{i}}{\partial q_{j}}\right|_{q}^{-1}=\sum_{q \in\left\{q^{\star}\right\}}\left|\prod_{i}\left(1-D_{+} \lambda_{i}\right)\right|_{q}^{-1},
$$

where $\left\{\boldsymbol{q}^{\star}\right\}$ is the set of Lagrangian locations solving for $\boldsymbol{x}$ in equation 1.1 , and $\lambda_{i}$ are the eigenvalues of the deformation tensor $d_{i j}$. Note that this expression for the density has singularities whenever for one of the eigenvalues we have

$$
\lambda_{i}=1 / D_{+}(t)
$$

This notion gives us the traditional interpretation of ZA, namely one where gravitational collapse occurs in three possible stages. First objects form along the major axis of collapse, making pancakes; then along the second eigenvector filaments form; and finally if and when all three eigenvalues have passed a singularity, a cluster forms. This 


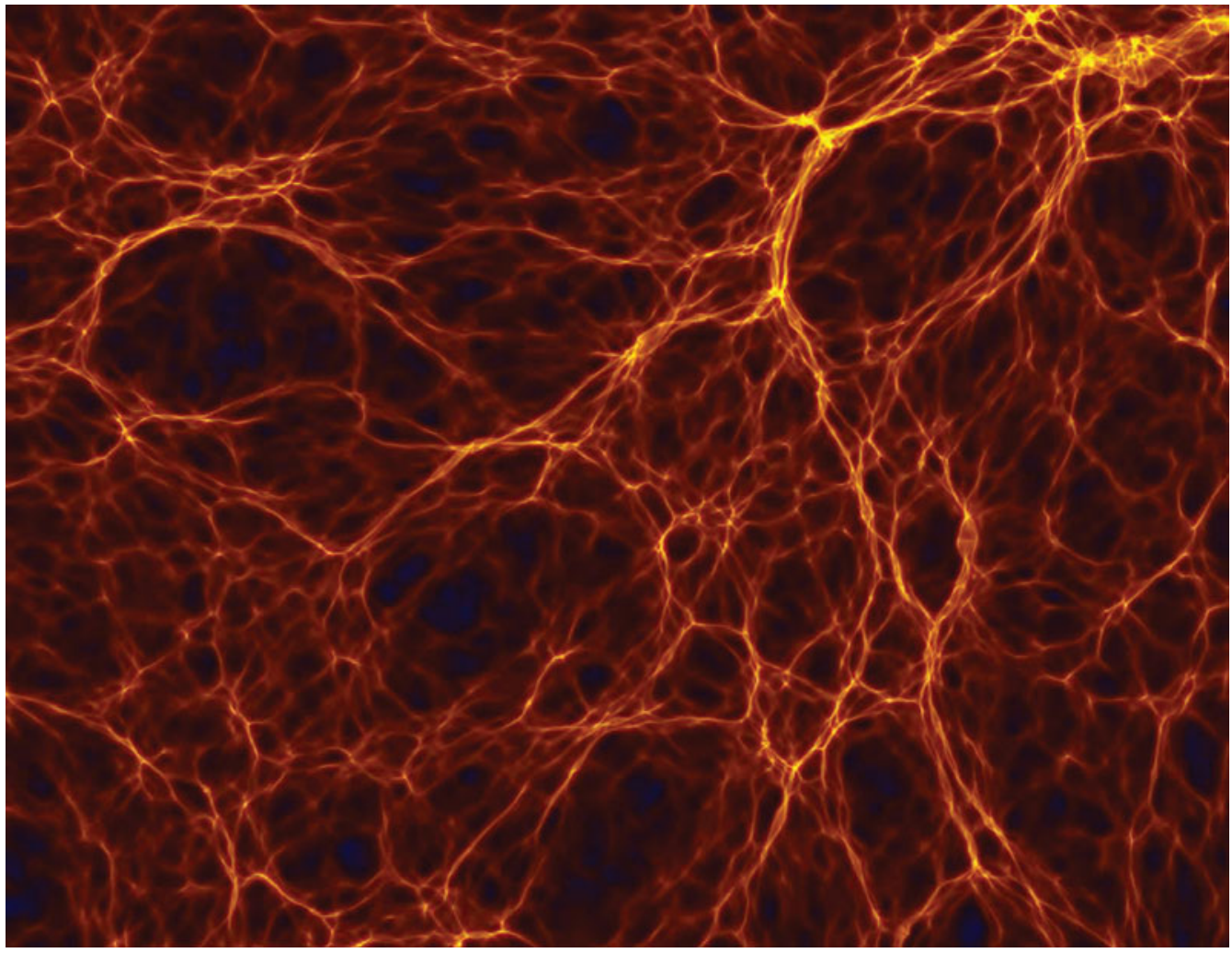

Figure 1. The Zeldovich approximation. Density map of the cosmic structure following the evolution according to the Zeldovich formalism. The cosmic web is sharply rendered, with most of the structures residing just before or around shell crossing. From Hidding 2016.

corresponds to the Morse theory view of nodes and saddles exploited in many structure finders. We show that, even in the narrow confines of the ZA, this interpretation is not complete; even that it is wrong on the account of the formation of the first filaments (Hidding et al. 2014). Taking the mathematics of Morse theory a step further, we arrive at Lagrangian singularity theory (Arnold et al. 1982, Arnold 1986). This theory shows how to predict the evolution of folds, cusps, swallow-tails, butterflies and umbilics directly from the initial velocity potential $\Phi_{0}$. Due to the relative complexity of this method we are forced to restrict our further discussion to the two-dimensional case.

Formation of pancakes. A fold is the simplest kind of singularity we have. It is the caustic that separates single-stream from multi-stream regions and is also found under the cryptic name $A_{2} \dagger$. At any moment in time, pancakes can be identified as the locations of $A_{2}$ folds. At a fold the phase-space sheet (see Fig. 2) is tangent to line of projection. In the case of ZA, this happens when $\lambda=1 / D_{+}$, identifying the level-sets of $\lambda$ as the Lagrangian progenitors of folds. Two folds may connect at a cusp. A fold being a line of tangency on the phase-space sheet, there exists points where the fold line itself is tangent to the projection, these points are the cusps (see Fig. 2). In the tensor field $d_{i j}$, a cusp is found where a level-set of $\lambda$ is tangent to the corresponding eigenvector $\boldsymbol{e}_{\lambda}$, or

$$
\nabla \lambda \cdot e_{\lambda}=0 .
$$

$\dagger A_{2}$ refers to the ADE classification of singularities introduced by Arnold. In this paper we also deal with $A_{3}$ for cusp, $A_{4}$ for swallow-tail, and $D_{4}$ for umbilic singularities. 

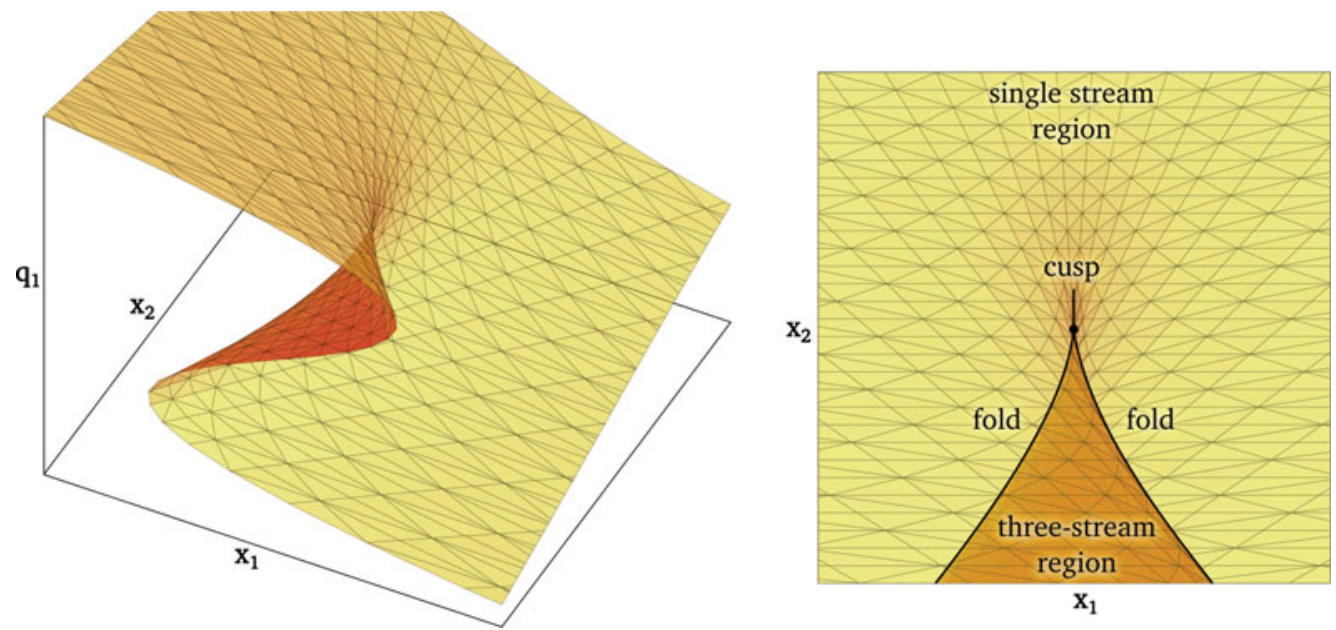

Figure 2. The cusp singularity. Singularities arise if we project a smooth manifold down one or more directions. In this case we show one Lagrangian direction as the $z$-axis and the two remaining axes in Eulerian space. On the right is the projected view, with the fold and cusp locations marked. Between the folds we find a three-stream region.
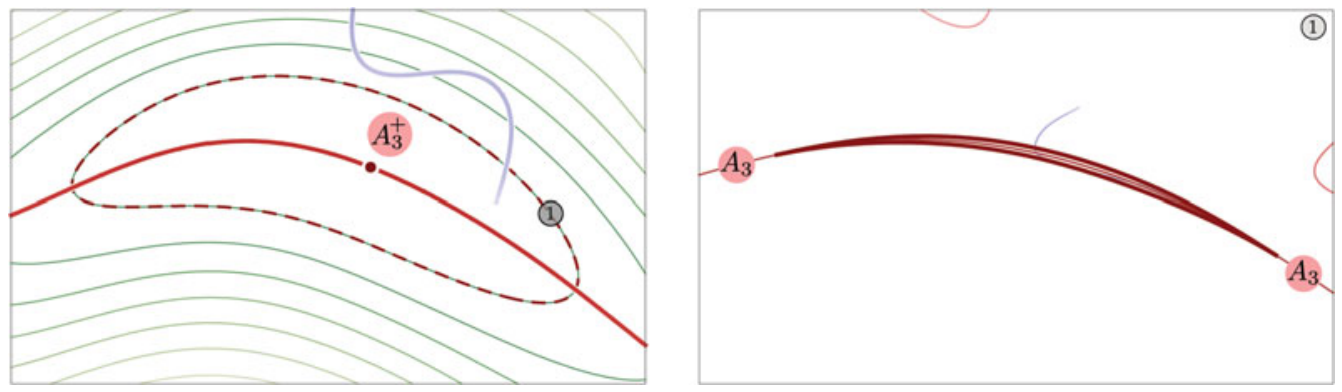

Figure 3. Pancake genesis. On the left we see in Lagrangian space the contours of the first eigenvalue around an $A_{3}^{+}$-point. The Eulerian counterpart of the dashed contour is shown on the right. It shows the pancake at its prime, highly elongated with a cusp on each end.

Finding all $A_{3}$-points for each level-set of $\lambda$ traces an $A_{3}$-line. The set of $A_{3}$-lines trace the entire network of filaments formed in the ZA, throughout time. It can be shown that all maxima and saddle points of the function $\lambda(\boldsymbol{q})$ also lie on an $A_{3}$-line. Lowering a levelplane down on the function $\lambda(\boldsymbol{q})$, we can see that at the maxima of $\lambda\left(A_{3}^{+}\right.$-points) two cusps are created, while at the saddle points $\left(A_{3}^{-}\right.$-points $)$they annihilate, merging two pancakes. $A_{3}$-lines terminate only in $D_{4}$ umbilic points, but we choose to also truncate them where $\lambda=0$, beyond which they loose their physical significance.

Splitting of pancakes. A pancake may branch by creating two new cusps at a fold. Typically one of these cusps remains within the confines of the present pancake and the other dashes out to create a subsidiary pancake, often to merge later with another pancake at an $A_{3}^{-}$-point, creating a three-legged structure. The point at which a pancake branches is called a swallow-tail, denoted $A_{4}$. An $A_{4}$ singularity is found in Lagrangian space at points where an $A_{3}$-line is tangent to the corresponding eigenvector, or equivalently at local maxima of $\lambda$ limited to the $A_{3}$-line. Important to note here, is that we don't need to involve the second eigenvalue to create a node in the network of caustics. Moving this discussion to the three-dimensional case, we don't strictly need to collapse along the 


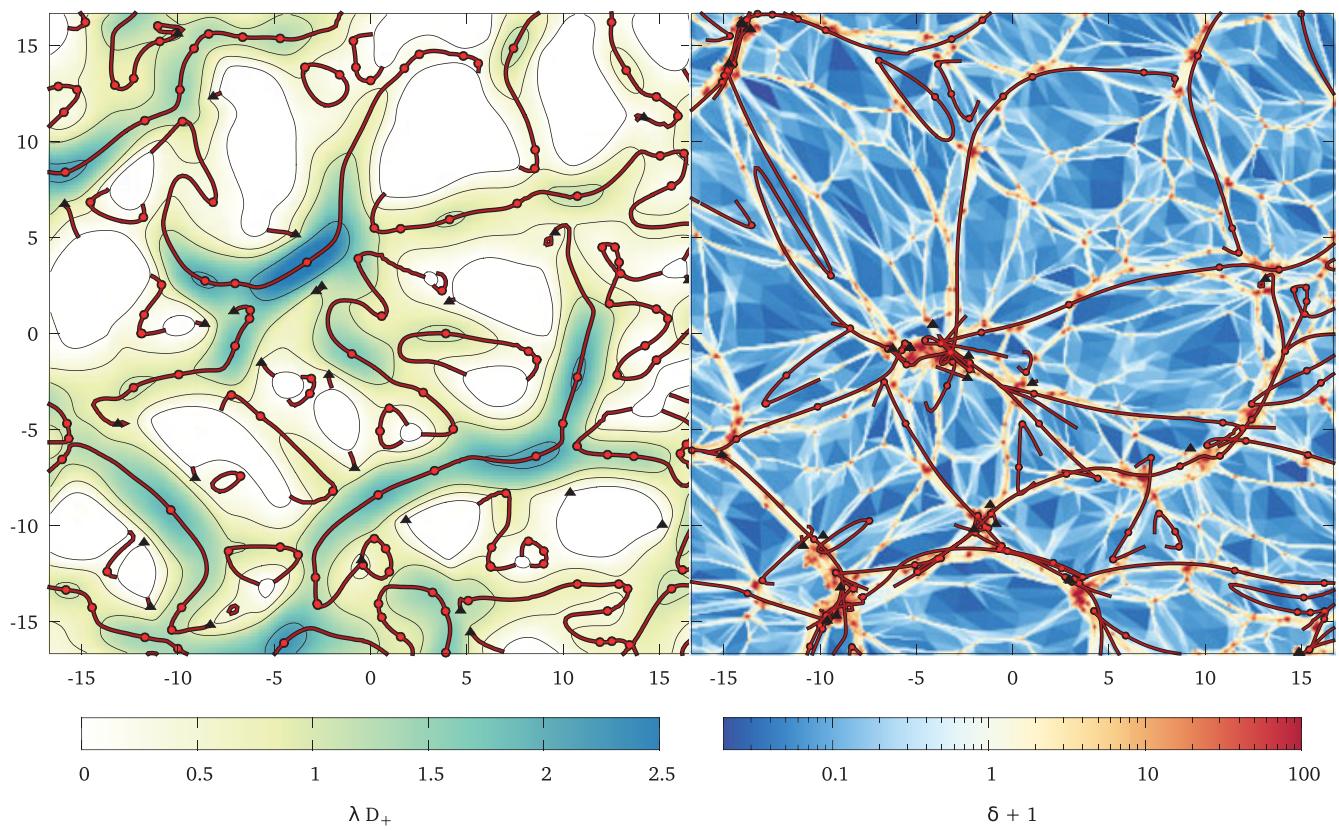

Figure 4. Comparison with $N$-body. On the left: ZA on filtered initial conditions; contours show the first eigenvalue, $A_{3}$-lines in red, and triangles showing $D_{4}$ points. On the right: the density contrast resulting from a 2D PM code, with the Eulerian displaced $A_{3}$-lines over-plotted.

second eigenvector to create a filament-like structure. This also suggests the existence of different possible late-time morphologies for filaments.

Scaling and comparison with $N$-body. We computed the $A_{3}$-lines for a set of initial conditions and compared the result with those of a 2D N-body code. A non-linear time evolution may be approximated by truncating power of the initial conditions at scales smaller than the scale of non-linearity. It is well known that observable filaments have a density contrast around unity, so this method should give realistic results. We find good agreement for all relevant $P(k) \propto k^{n}$ power-spectra, in an eye-ball comparison of filaments predicted by ZA with density fields from a 2D PM code. An example is given in Fig. 4.

\section{The Adhesion approximation}

We showed how the emergence of caustics in the ZA allows us to trace the formation of cosmic structures in a formal, yet physically meaningful way. However, the ZA suffers from a major flaw in that it doesn't allow for gravitational interaction, and therefore hierarchical structure formation. This is because the ZA is solely based on local analysis of the velocity potential and its chain of derivatives. The adhesion model moves beyond local considerations, which makes it computationally more intensive than the ZA (though still much faster than N-body). Still, results are computed from initial conditions directly and with complete accountability. The adhesion model is arrived at by taking the source-free (hence collision-free) Euler equation, and adding an artificial viscosity term to emulate the effects of gravity (Gurbatov \& Saichev 1984, Shandarin \& Zeldovich 1989). The resulting equation

$$
\partial_{t} \boldsymbol{u}+(\boldsymbol{u} \cdot \nabla) \boldsymbol{u}=\nu \nabla^{2} \boldsymbol{u}
$$



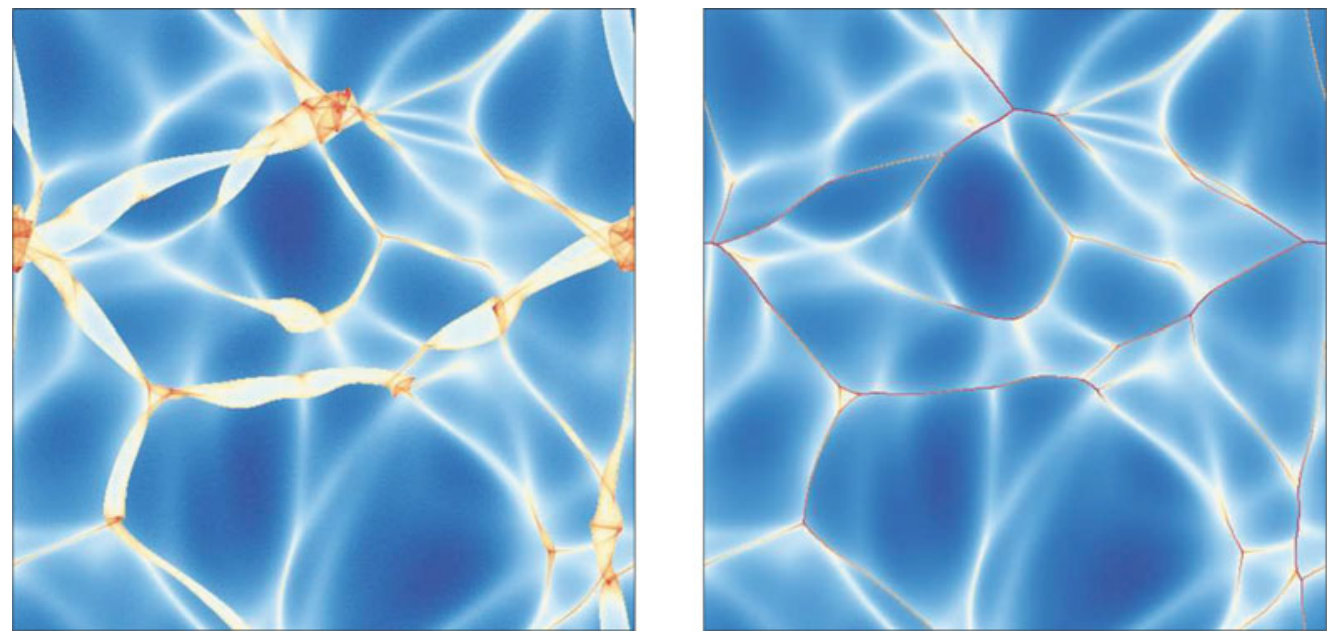

Figure 5. ZA and adhesion compared. Outside multi-stream regions the results from the ZA and adhesion are identical. Adhesion contains an artificial viscosity term that only 'activates' when streams cross. Multi-stream regions are thus collapsed to infinitesimally thin structures.
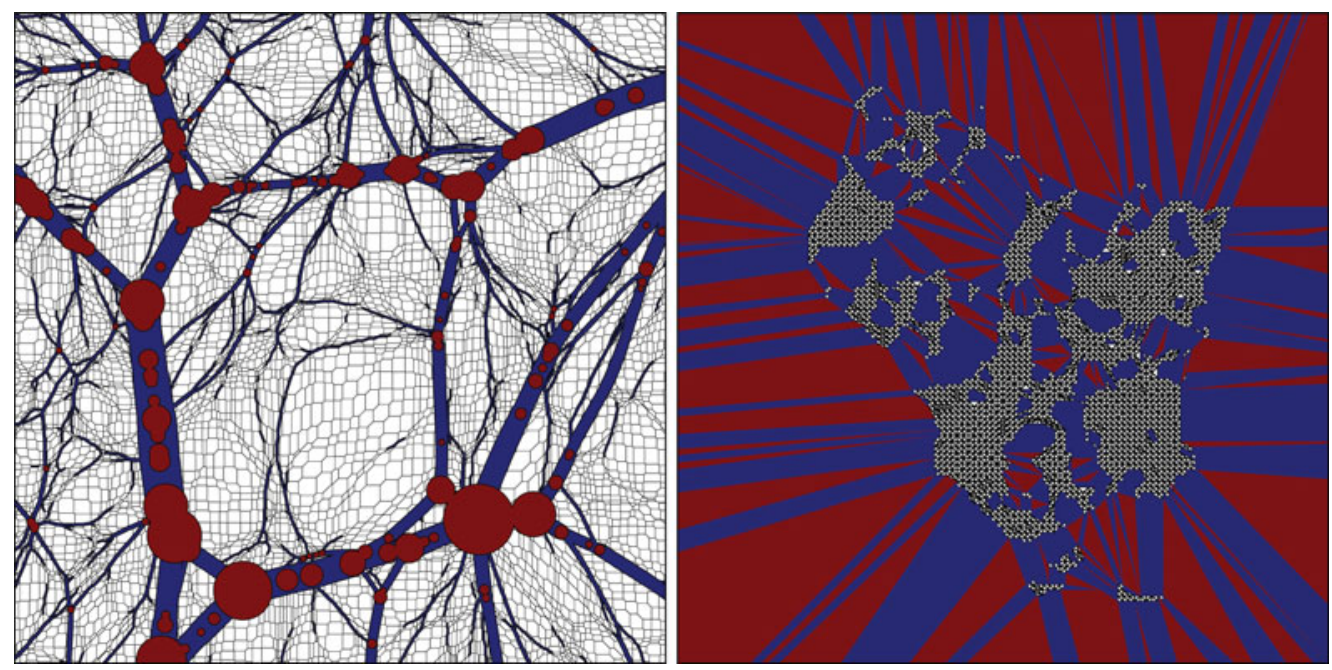

Figure 6. Dual structures in adhesion.

is known as Burgers' equation; in the limit where $\nu \rightarrow 0$, it has the exact solution

$$
\Phi(\boldsymbol{x}, t)=\max _{q}\left(\Phi_{0}(\boldsymbol{q})-\frac{(\boldsymbol{x}-\boldsymbol{q})^{2}}{2 D_{+}(t)}\right) .
$$

The global maximum in this solution can be computed efficiently using either a Legendre transform, convex hull (Vergassola et al. 1994) or a weighted Voronoi diagram (Hidding et al. 2012, Hidding et al. 2016a (2016)). One condition for reaching an extremum is that the first derivative of the maximised quantity should vanish. Performing this test reduces above equation to the $\mathrm{ZA}$ as presented in equation 1.1. The global maximisation guarantees that the resulting map from Lagrangian to Eulerian space stays monotonic always. Where and whenever shell-crossing occurs in the ZA, adhesion creates a solid structure. Matter inside these structures is confined to stay inside, which is the reason people may refer to the adhesion model as having "sticky particles". Outside collapsed 


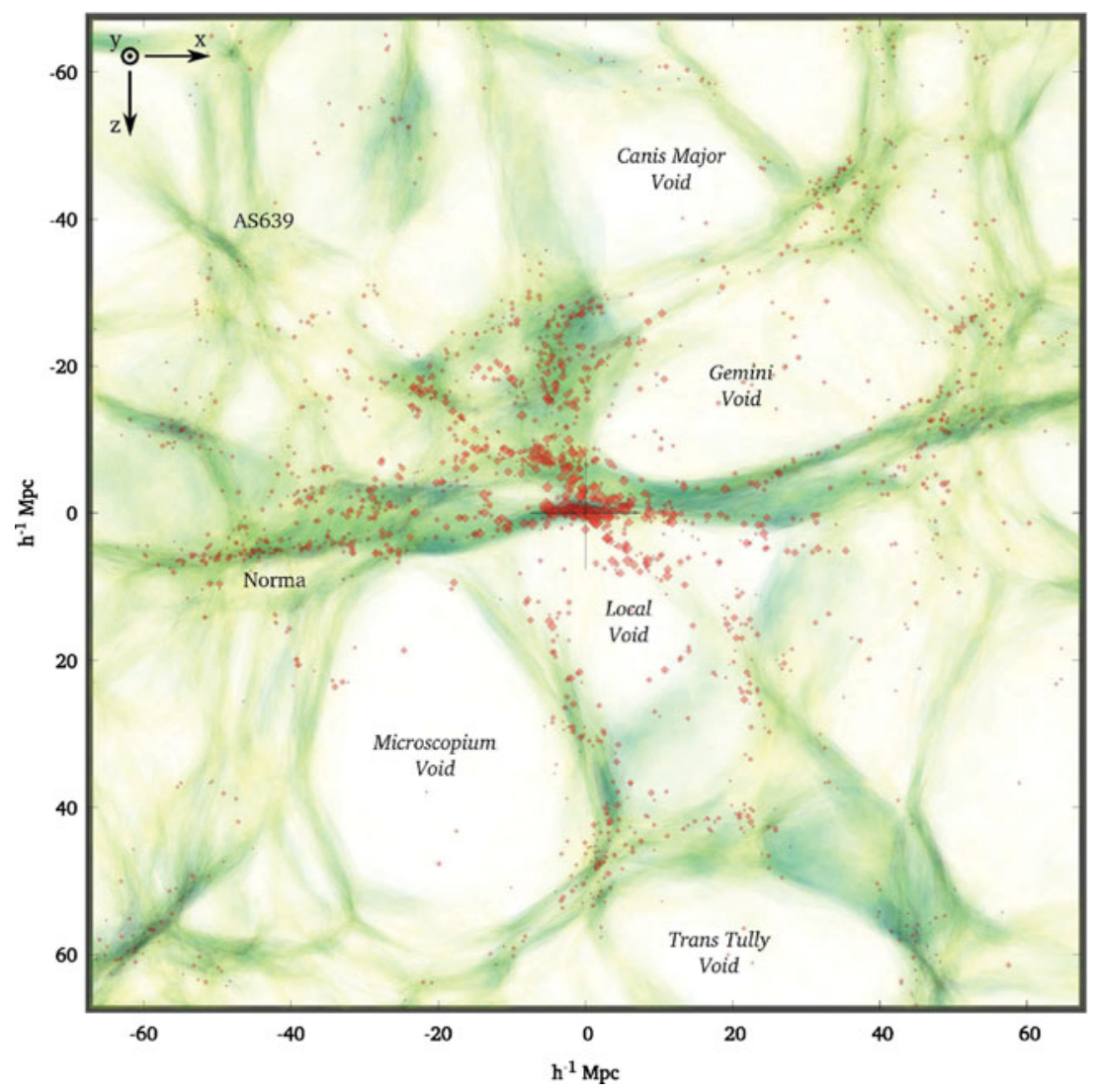

Figure 7. Local Universe adhesion reconstruction. Structure of the lcoal Universe. The reconstruction of the weblike structure in the local Universe, sampled by the 2MRS survey, has been obtained on the basis of the adhesion formalism applied to a set of 25 constrained Bayesian KIGEN realizations of the primordial density and velocity field in the Local Universe. The image shows the density field in a $10 \mathrm{Mpc}$ thick slice perpendicular to the plane of the Local Supercluster. Note that the density field concerns the dark matter distribution. The red dots are the 2MRS galaxies in the same volume. From Hidding 2016, Hidding et al. 2016b.

structures the results from the ZA and adhesion are identical; caustics from ZA are compressed to infinitesimally thin structures (see Fig. 5). This unifies Zeldovich' idea of collapsed structures in terms of shell crossing with a hierarchical formation model.

Dual geometry. The solution to Burgers' equation given in expression 2.2 is identical to the definition of the weighted Voronoi tessellation, weighted by the potential. The Voronoi cell of a Lagrangian point $\boldsymbol{q} \in \mathcal{L}$ occupies an area in Eulerian space $\mathcal{E}$ given by

$$
V_{q}=\left\{\boldsymbol{x} \in \mathcal{E} \mid(\boldsymbol{x}-\boldsymbol{q})^{2}+w_{q} \leqslant(\boldsymbol{x}-\boldsymbol{p})^{2}+w_{p}, \forall \boldsymbol{p} \in \mathcal{L}\right\}
$$

Taking $w(\boldsymbol{q})=2 D_{+} \Phi_{0}(\boldsymbol{q})$ as the weights in this expression reduces it to the given solution in equation 2.2. Where there is a Voronoi tessellation, there is its dual: the Delaunay triangulation. It is the latter that gives us the origin and mass of matter residing in the nodes, edges and faces of the Voronoi tessellation (see Fig. 6). 


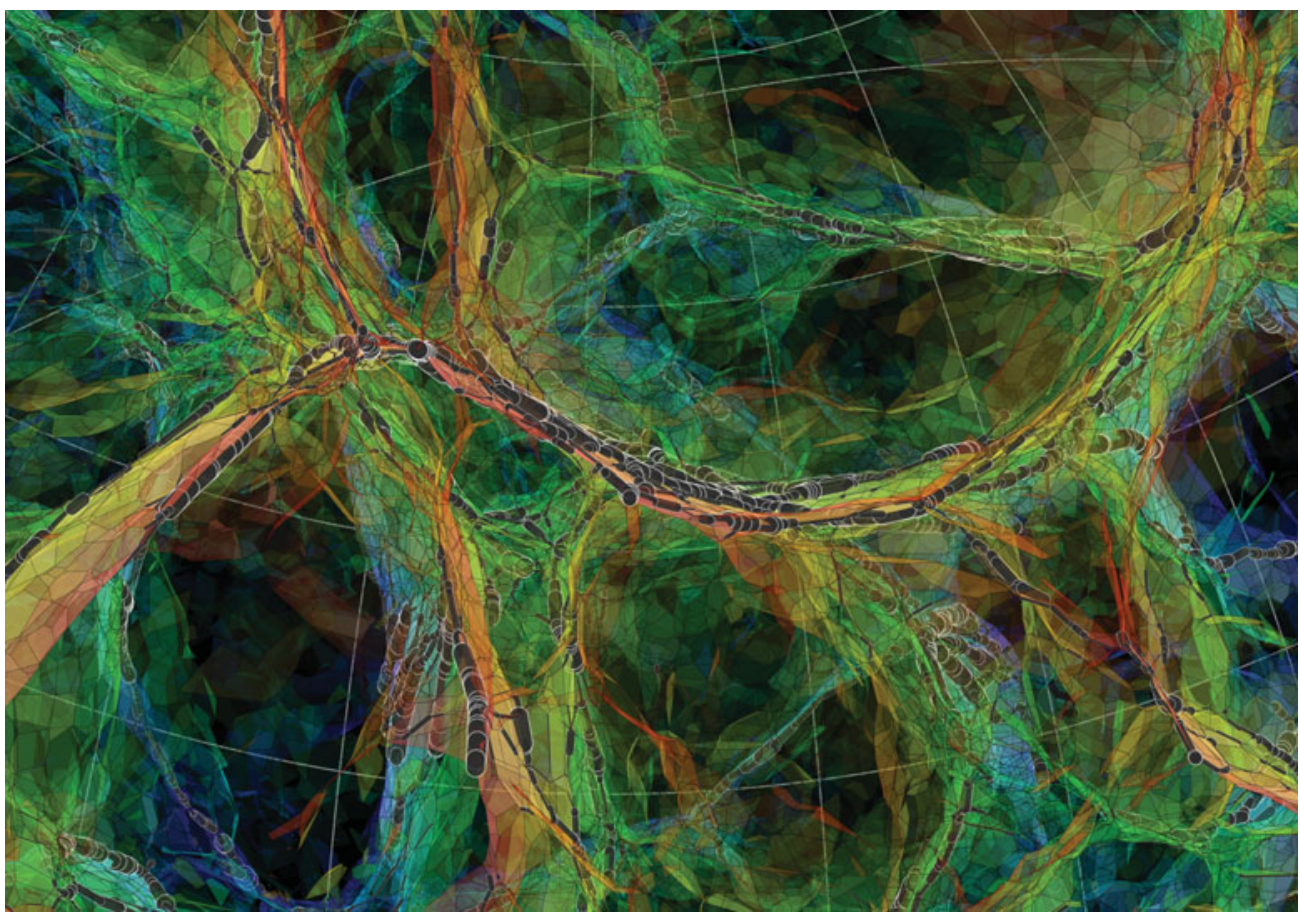

Figure 8. The Pisces-Perseus Supercluster. A 3-D isodensity surface rendering of the intricate filamentary structure around the Pisces-Perseus supercluster. It is based on the adhesion reconstruction of the local Cosmic Web, based on constrained realizations of the local primordial density and velocity field implied by the 2MRS galaxy redshift survey. From Hidding 2016, Hidding et al. 2016b.

\section{The Local Universe}

One application of the adhesion model is the detection of walls, filaments and nodes in cases where some form of an initial potential is available. We ran our adhesion code on a set of 25 constrained initial conditions reconstructed (Kitaura 2013, Heß et al. 2013) to produce structures in our local universe $(z<0.03)$ (Hidding 2016, Hidding et al. $2016 \mathrm{~b}$ (2016)). This reconstruction is based on the 2MASS redshift catalog (Huchra et al. 2012), which covers the full sky except for galactic lattitudes $|b|<5^{\circ}$.

Figure 7 provides a remarkably detailed reconstruction of the cosmic web in the $2 \mathrm{MRS}$ volume. It shows the (surface) density of the weblike structures in the Local Universe. These are the result of adhesion simulations by Hidding 2016 and Hidding et al. 2016b (2016), based on the the constrained Bayesian KIGEN reconstruction by Kitaura 2013 of the initial conditions in the local volume traced by the 2MRS redshift survey. For a given Gaussian primordial field, the adhesion formalism allows the accurate reconstruction of the rich pattern of weblike features that emerge in the same region as a result of gravitational evolution. The adhesion formalism was applied to 25 constrained realizations of the 2MRS based primordial density field (Hidding et al. 2012, Hidding 2016). The mean of these realizations gives a reasonably accurate representation of the significant filamentary and wall-like features in the Local Universe. Most outstanding is the clear outline of the void population in the local Universe. The reconstruction also includes the velocity flow in the same cosmic region. It reveals the prominent nature of the outflow from the underdense voids, clearly forming a key aspect of the dynamics of the Megaparsec scale universe. 
The Local Universe structure in figure 7 presents a telling image of a void dominated large scale Universe. Many of the voids in the adhesion reconstruction can be identified with the void nomenclature proposed by Fairall (Fairall (1998)), who mainly identified these voids by eye from the 6dFGRS survey. It is interesting to see that the socalled Tully void appears to be a richly structured underdense region, containing at least the Microscopium Void, the Local Void and the "Trans Tully Void".

In the same reconstruction, we are studying the intricate filamentary network in and around the Pisces-Perseus supercluster. The image in figure 8 provides a nice impresssion of the complex 3-dimensional structure and connectivity along the main ridge of the Pisces-Perseus supercluster. It also shows how the main ridge connects to several neighbouring filaments, connecting near massive clusters along the ridge, and how these filaments surround a lower density planar structure. Interesting is to note the clustering and alignment of the small (whitish) filamentary tendrils in and around the main arteries of the Cosmic Web. Analysis of this weblike structures region is under progress and will be first reported in Hidding 2016.

\section{References}

Abel, T., Hahn, O., \& Kaehler, R. 2012, MNRAS, 427, 61-76

Arnold, V. I. 1986, Journal of Soviet Mathematics, 32:03, 229-258

Arnold, V. I., Shandarin, S. F., \& Zeldovich, Ya. B. 1982, Geophysical and Astrophysical Fluid Dynamics, 20, 111-130

Fairall A. P. 1998, Large-scale structures in the Universe (Wiley)

Falck, B. L., Neyrinck, M. C., \& Szalay, A. S. 2012, ApJ, 754, 126

Gurbatov, S. N. \& Saichev, A. I. 1984, Radiophysics and Quantum Electronics, 27:4, 303-313

Heß, S., Kitaura, F.-S., \& Gottlöber, S. 2013, MNRAS 435, 2065-2076

Hidding, J., Van de Weygaert, R., Vegter, G., Jones, B. J. T., \& Teillaud, M. 2012, Proc. of the 28th SoCG, doi:10.1145/2261250.2261316

Hidding, J., Shandarin, S. F., \& Van de Weygaert, R. 2014, MNRAS, 437, 3442-3472

Hidding, J., 2016, in preparation $\mathrm{PhD}$ thesis, Univ. Groningen

Hidding, J., Van de Weygaert, R., \& Vegter, G. 2016, in preparation.

Hidding, J., Van de Weygaert, R., Kitaura F.-S. \& Hess S. 2016, in preparation.

Huchra, J. P., Macri, L. M., Masters, K. L. et al. 2012, ApJS, 199, 26

Kitaura, F.-S. 2013, MNRAS, 429, L84-L88

Shandarin, S. F. \& Zeldovich, Ya. B. 1989, Rev. Mod. Phys., 61, 185-220

Shandarin, S. F., Habib, S., \& Heitmann, K. 2012, Phys. Rev. D, 85, 083005

Vergassola, M., Dubrulle, B., Frisch, U., \& Noullez, A. 1994, A\& A, 289, 325-356

Zeldovich, Ya. B. 1970, $A \mathscr{G} A, 5,84-89$ 